\title{
SIGS - Seismic Inferences for Glitches in Stars
}

\author{
L. Filipe R. Pereira ${ }^{1,2}$, João P. S. Faria ${ }^{1,2}$, and Mário J. P. F. G. Monteiro ${ }^{1,2}$ \\ ${ }^{1}$ Departamento de F'isica e Astronomia, Faculdade de Ciências da Universidade do Porto, Portugal \\ ${ }^{2}$ Instituto de Astrofísica e Ciências do Espaço, Universidade do Porto, Portugal
}

\begin{abstract}
The increased amount of high precision seismic data for solar-like stars calls for the existence of tools that can extract information from such data. In the case of the study of acoustic glitches there are no publicly available tools and most of the existing ones require a deep knowledge of their implementation. In this work a tool is presented that aims to both simplify the interaction with the user and also be capable of working automatically to determine properties of acoustic glitches from seismic data of solar-like stars. This tool is shown to work with both the Sun and other solar analogs but there are still some limitations to the methods being used when considering smaller frequency datasets.
\end{abstract}

\section{Introduction}

Acoustic glitches, such as the base of the convective zone (therein $\mathrm{BCZ}$ ) and the helium second ionization zone (therein HeII), are regions in the interior of a star where the sound speed suffers an abrupt variation due to the sharp change in the internal structure. Since the eigenfrequencies of a star are directly related to the local sound speed, these glitches cause perturbations in thoem. These perturbations exhibit an oscillatory behavior when considering frequencies of sequential radial order [1-3]. It is thus possible to characterize some properties of these acoustic glitches by isolating and studying their contribution to the eigenfrequencies.

This oscillatory signature from the acoustic glitches has been extensively studied for the Sun to determine the location of the two previosly mentioned acoustic glitches, BCZ and HeII $[4,5]$ and it has also been applied to solarlike stars [6-9].

However, the existing tools to perform this study are not publicly available and require an intimate knowledge of the implemented methods in order to achieve good results. The work presented here aims to improve upon existing methods in order to make available a numerical procedure that is both more precise than the current methods and at the same time highly user-friendly so that it can be openly distributed to the community. Starting from the work developed by Faria [8], which in turn was adapted from the works of Monteiro et al. [4], Monteiro and Thompson [5] and Mazumdar et al. [9] the method was improved upon and an automatic pipeline was implemented to achieve robust results with minor user intervention.

The presented tool is validated initially by using observational data from the Sun and afterwards it is applied to both stars from the 16 Cygni binary and also to 10 solarlike stars chosen from the sample of Mazumdar et al. [9].

In the next section both methods implemented in the tool are described. Then in section 3 the code is validated using data from the Sun. In section 4 the automatic implementations of the code are described. In section 5 the results obtained for the selected stars are shown and finally in section 6 the results are discussed and some conclusions are drawn.

\section{Methods}

The code presented in this work is comprised of two similar but independent methods capable of isolating the oscillatory signature of the acoustic glitches and then determining some of their properties.

\subsection{Method A - Frequencies}

Method A isolates the signature in the frequencies themselves by iteratively removing a smooth component from the frequencies and then fitting the residuals to the free parameters from equation

$$
\begin{aligned}
\delta v & \simeq v_{s}+ \\
& +A_{\mathrm{BCZ}}\left(\frac{v_{r}}{v}\right)^{2} \cos \left(4 \pi \tau_{\mathrm{BCZ}} v+2 \phi_{\mathrm{BCZ}}\right)+ \\
& +A_{\mathrm{HeII}}\left(\frac{v_{r}}{v}\right) \sin ^{2}\left(2 \pi \beta_{\mathrm{HeII}} v\right) \cos \left(4 \pi \tau_{\mathrm{HeII}} v+2 \phi_{\text {HeII }}\right),
\end{aligned}
$$

adapted from Faria [8] where $A_{\mathrm{BCZ}}, \tau_{\mathrm{BCZ}}$ and $\phi_{\mathrm{BCZ}}$ correspond, respectively, to the amplitude, acoustic depth and phase of the signal from the base of the convective zone. While $A_{\text {HeII }}, \tau_{\text {HeII }}, \phi_{\text {HeII }}$ and $\beta_{\text {HeII }}$ correspond, respectively, to amplitude, acoustic depth, phase and finally, the acoustic width of the signal, in this case due to the helium second ionization zone. The frequency $v_{s}$ is the smooth component removed from the frequencies. It is obtained independently for the $N_{l}$ frequencies of each angular degree $l$ 


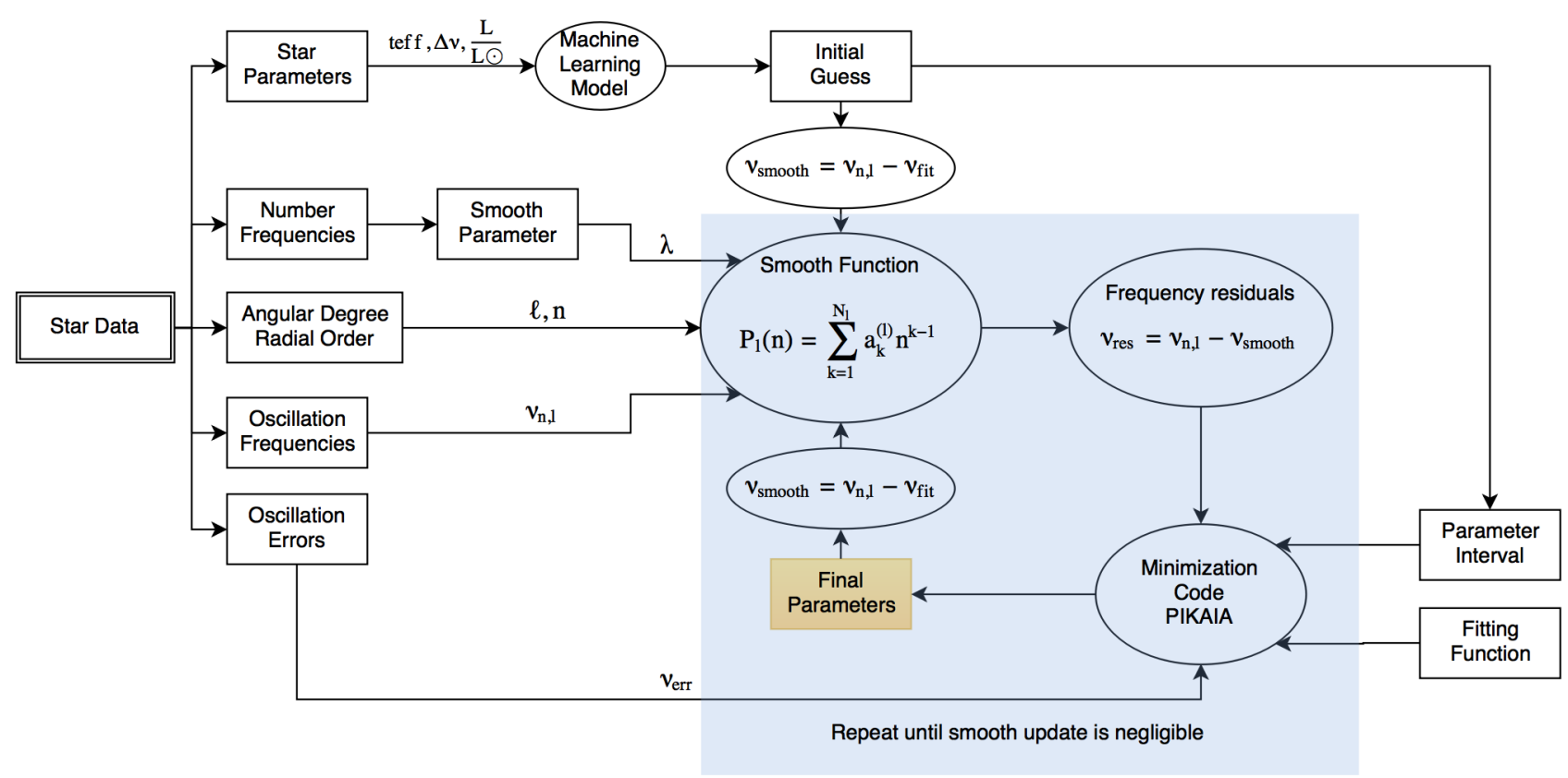

Figure 1: Diagram of the workflow of method A in SIGS, which determines the acoustic depths of the base of the convective zone and helium II ionization zone of a star by extracting the signal caused by these glitches directly from the oscillation frequencies of the star.

by fitting a polynomial of degree $N_{l}-1$ with third derivative smoothing [4]. This smoothing can be controlled by defining a smoothing parameter $\lambda$, which can be selected by the user. By default, this parameter can be determined automatically in the code.

After removing the smooth function from the frequencies, the residuals are fitted to Equation 1 using the PIKAIA genetic algorithm [10] which is a global minimization algorithm that utilizes concepts inspired by the processes of evolution by natural selection. This method is advantageous compared to hill climbing methods because it doesn't require an initial guess and instead it explores the user-defined range of possible values for each of the free parameters and should, given enough time, converge to global minimum in the parameter space. Minimization is achieved by initiating a population of sets of possible solutions chosen randomly from the interval and then evolving then for a specified number of generations. Through the generations the best sets from the population are chosen to move forward by using a standard chi squared fitness function and after the evolution process the best set of parameters is chosen as the correct one.

The process of executing the smooth removal and fitting the residuals to the equation is repeated until the smooth function is perfectly characterized and convergence is said to be achieved. Figure 1 shows a diagram of the general workflow of this method

\subsection{Method B - Second differences}

In method B, instead of using the frequencies, the method searches for the signature of the glitches in the second differences [3]. In this method the smooth component is only removed once and the functional form of this component is a polynomial of a degree up to three depending on the amount of data points available for the fit.

After removing the smooth function the procedure is similar to the other method with the exception that there is no iteration on the smooth component and the functional form of the signal is taken to be

$$
\begin{aligned}
\delta \Delta_{2} v & \simeq \Delta 2 v_{s}+ \\
+ & A_{\mathrm{BCZ}}^{*}\left(\frac{v_{r}}{v}\right)^{2} \sin \left(4 \pi \tau_{\mathrm{BCZ}} v+2 \phi_{\mathrm{BCZ}}\right)+ \\
+ & A_{\mathrm{HeII}}^{*}\left(\frac{v}{v_{r}}\right) \exp \left[-\beta_{\mathrm{HeII}}^{*}\left(\frac{v_{r}}{v}\right)^{2}\right] \sin \left(4 \pi \tau_{\mathrm{HeII}} v+2 \phi_{\text {HeII }}\right)
\end{aligned}
$$

which is very similar to the one from the frequencies, with the same seven free parameters, but a different smooth function as mentioned previously. This expression was adapted from the work of Mazumdar et al. [9], which in turn was adapted from the work of Houdek and Gough [7]. Figure 2 shows the workflow of method B.

\subsection{Monte Carlo Simulations}

To add robustness to both methods and estimate the errors in the measurements of the parameters we perform Monte Carlo simulations. We generate alternative data sets by taking the original frequencies and changing them by a value sampled from a normal distribution with standard deviation equal to the uncertainty of the original frequency. The methods are then used on all the new data sets to obtain a distribution of values for the final parameters.

\section{Validation}

To validate the implementations of both methods we apply them to both low-degree solar frequencies [11] and then 


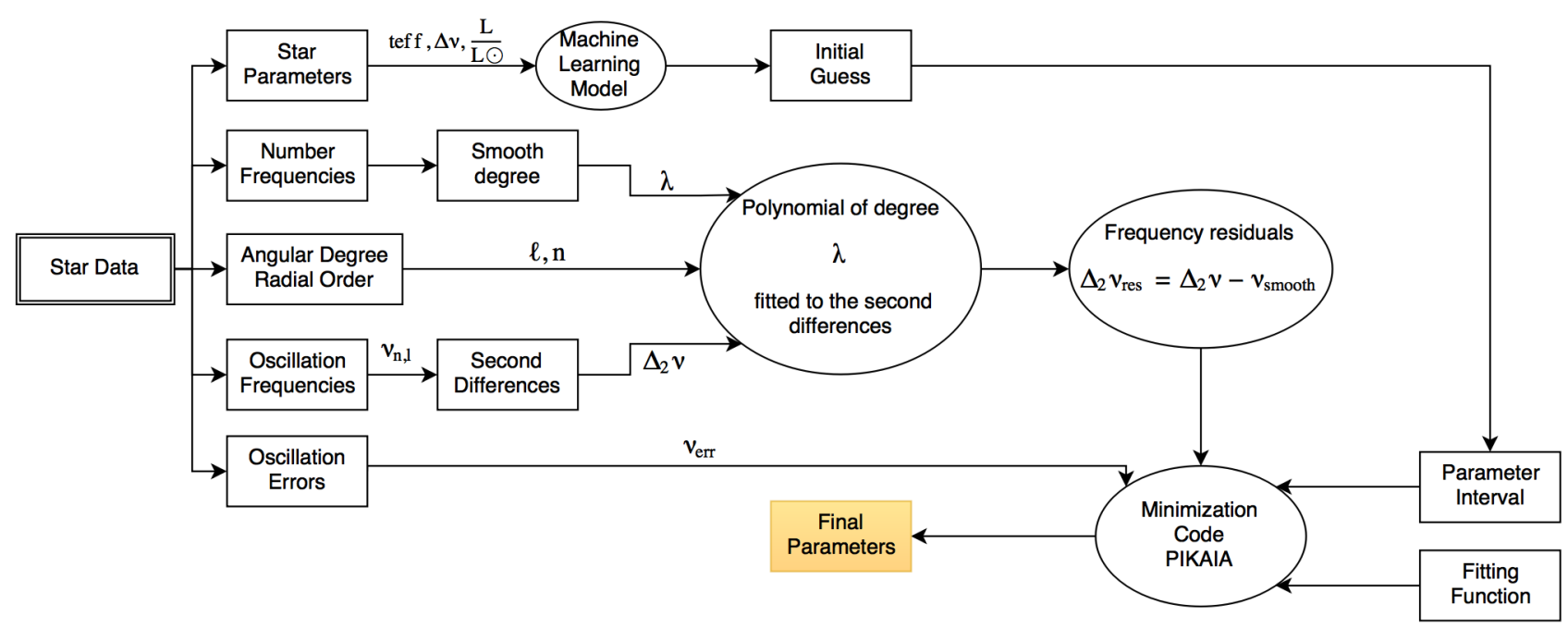

Figure 2: Diagram of the workflow of method B in SIGS, which determines the acoustic depths of the base of the convective zone and helium II ionization zone of a star by extracting the signal caused by these glitches from the second differences of the frequencies of the star.

compare the results with that of previous works. The results obtained for both methods are summarized in Table 1.

Table 1: Comparison of the acoustic location of the Sun's acoustic glitches, determined using observational data.

\begin{tabular}{c|cc|cc}
\hline & \multicolumn{2}{|c}{ This Work } & \multicolumn{2}{c}{ Previous Works } \\
& $\delta v$ & $\Delta 2 v$ & $\delta v$ & $\Delta 2 v$ \\
\hline$\tau_{\mathrm{BCZ}}(s)$ & 2282 & 2314 & 2337 & 2273 \\
$\tau_{\mathrm{HeII}}(s)$ & 685 & 692 & 649 & 707 \\
\hline
\end{tabular}

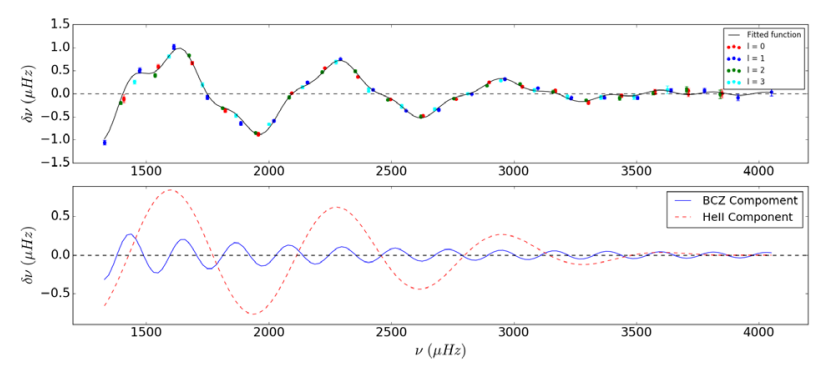

Figure 3: The top panel shows the fit of the residuals from the solar frequencies to Equation 1 using Method A. The color scheme detailed in the legend is used to differentiate between the degrees $l$ of the frequencies. The bottom panel has the signal components from both the BCZ and HeII resulting from the fitting of the upper panel.

Figures 3 and 4 show fits to the best parameters and the oscillatory signature of both glitches for methods A and B respectively. These show how the code is working successfully in isolating the signals. The small difference in values compared to the literature is to be expected, considering the small changes made to the methods implemented and to the more recent data used.

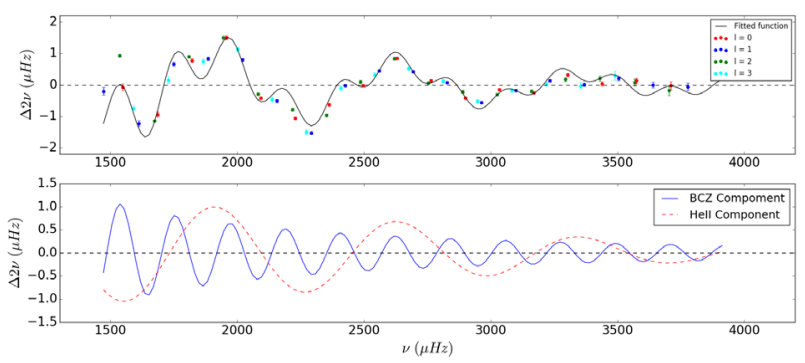

Figure 4: The top panel shows the fit of the second differences from the solar frequencies to Equation 2 using Method B. The color scheme detailed in the legend is used to differentiate between the degrees $l$ of the second differences. The bottom panel has the signal components from both the $\mathrm{BCZ}$ and $\mathrm{HeII}$ resulting from the fitting of the upper panel.

\section{Automatic Pipeline}

After validating the code using the Sun, the program was used to determine the parameters of a grid of models and the results were used to build a pipeline that was capable of reducing the parameter interval automatically to improve robustness to the results without requiring user interaction.

A grid [12] of CESAM [13] models was considered with masses between $0.8 M_{\odot}$ and $1.2 M_{\odot}$ in intervals of $0.04 M_{\odot}$ and each mass was considered with 5 different ages $(1.5,3,4.5,6$ and $8 \mathrm{Gyr})$. The frequencies of the models were computed using the POSC Code [14]. The results from this run were then used to associate the determined values of the acoustic depths of both glitches to the values of effective temperature $\left(T_{\text {eff }}\right)$ and large frequency separation (Large_sep) of the stars through a multivariate quadratic regression. The result of the regression is shown in Figure 5. Only the results using method A were shown, since the results for method B are very similar. 

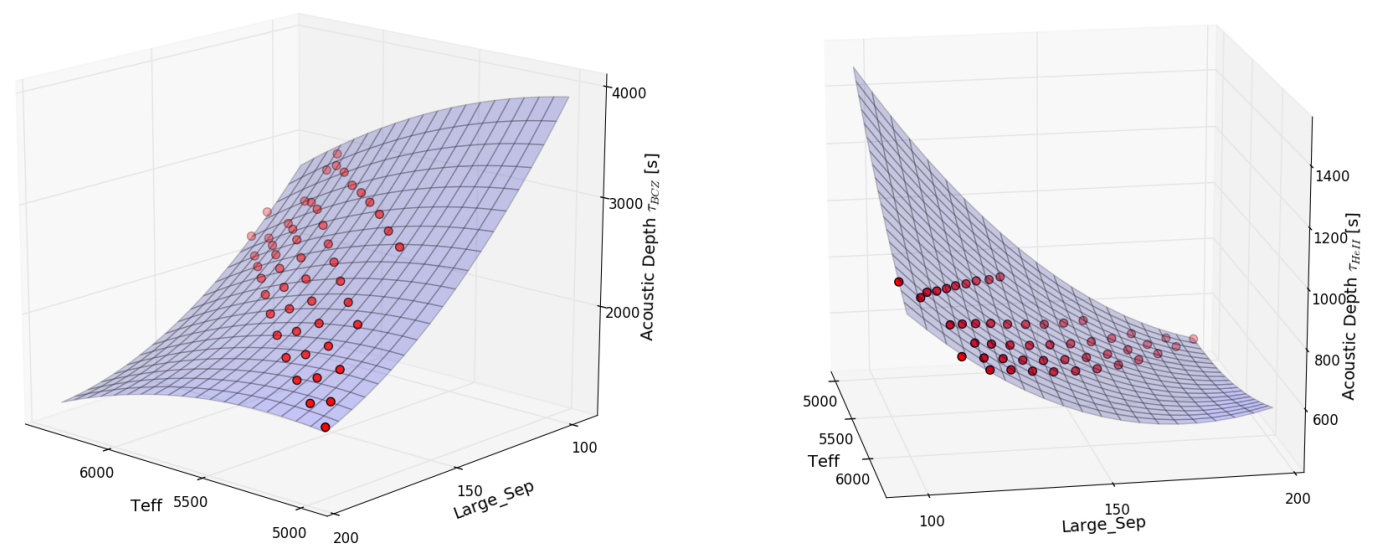

Figure 5: Multivariate regression applied to the values of effective temperature $\left(T_{\text {eff }}\right)$, and large frequency separation (Large_Sep), to the values of acoustic depth of the base of the convective zone (on the left) and the helium second ionization zone (on the right). The blue surface is the quadratic plane fitted.

The results from this regression allowed for the definition of a function that estimates the value of the acoustic depths of both glitches from the $T_{\text {eff }}$ and Large_Sep of a star, improving the focus (and convergence) of the fitting procedure.

\section{Results}

To test the complete tool, which is to be available to the community, the program was applied to the stars of the 16 Cygni binary, with data from Verma et al. [15], and to 10 solar-like stars from the sample of Mazumdar et al. [9] with frequencies from Appourchaux et al. [16]. The errors determined for the results were obtained by executing the program for 500 realizations of the observational data of each star, using the aforementioned Monte Carlo simulations. The results for $16 \mathrm{Cyg} \mathrm{A}$ and $\mathrm{B}$ are presented in Table 2. Table 3 shows the results for the 10 selected stars.

Table 2: Results obtained in each method for 16 Cyg A (on the top) and 16 Cyg B (on the bottom).

\begin{tabular}{ccc}
\hline 16 Cyg A & Method A & Method B \\
\hline$\tau_{\mathrm{BCZ}}(s)$ & $2904 \pm 89$ & $3038 \pm 148$ \\
$\tau_{\mathrm{HeII}}(s)$ & $1178 \pm 40$ & $982 \pm 14$ \\
\hline
\end{tabular}

\begin{tabular}{ccc}
\hline 16 Cyg B & Method A & Method B \\
\hline$\tau_{\mathrm{BCZ}}(s)$ & $2538 \pm 263$ & $2452 \pm 243$ \\
$\tau_{\mathrm{HeII}}(s)$ & $947 \pm 31$ & $902 \pm 31$ \\
\hline
\end{tabular}

\section{Discussion and Conclusions}

This work presents a Code, to be made publicly available, that implements two alternative methods to measure the signature of acoustic glitches in the low-degree oscillation data of solar-like stars. The code was validated using solar seismic data and tested on an application to a sample of solar type stars with seismic data available from Kepler [16].

For the two components of the 16 Cygni stellar binary, the results reported here show precise parameters that are very similar to the ones found in other works [15]. For the 10 selected stars from [15], the results are compatible with the previous measurements in [9], although for some stars the code did not converge with any of the two methods.

The lack of convergence is most likely related to the amount of available data points for some of these 10 stars, and not to the procedures that were implemented, as these work very well for stars with a higher number of data points (either frequencies for method $\mathrm{A}$ or second differences for method B). This is a difficulty that has already be found with a the manual implementation of the methods (see [15]), which becomes worse when an automatic procedure is adopted, as done in this work. Indeed, the results reported here confirm that with the automatic pipeline, that reduces the parameter space for the fitting algorithm, the methods are still highly dependent on the amount and quality of the available data points.

The results for the Sun and the stars of the 6 Cygni binary show that SIGS is a tool with potential to be used in large amount of data sets, as it achieves the same results as other methods without the need for user intervention and guidance. Some improvements of the code are possible (and some are already ongoing regarding convergence). It will also be quite simple to add additional fitting functions (and number of free parameters) for the signature of the glitches in order to constrain the signal over a wider range of stellar masses and ages. Such a tool may be relevant for 
Table 3: Results obtained from both methods for the 10 selected solar-like stars.

\begin{tabular}{c|c|c|c|c}
\hline \multirow{2}{*}{ Star } & \multicolumn{2}{|c}{ Method A } & \multicolumn{2}{c}{ Method B } \\
& $\tau_{\text {BCZ }}(s)$ & $\tau_{\text {HeII }}(s)$ & $\tau_{\text {BCZ }}(s)$ & $\tau_{\text {HeII }}(s)$ \\
\hline KIC008006161 & $2140 \pm 69$ & $613 \pm 27$ & $2234 \pm 51$ & - \\
KIC008379927 & - & $969 \pm 31$ & - & $841 \pm 73$ \\
KIC008760414 & - & - & - & - \\
KIC006603624 & - & $1084 \pm 52$ & $3066 \pm 213$ & $936 \pm 50$ \\
KIC010454113 & - & $838 \pm 10$ & $2599 \pm 90$ & $751 \pm 20$ \\
KIC006106415 & - & - & - & $963 \pm 81$ \\
KIC010963065 & $2853 \pm 101$ & $1006 \pm 33$ & $2792 \pm 139$ & $1003 \pm 73$ \\
KIC006116048 & - & $1166 \pm 36$ & - & $1071 \pm 81$ \\
KIC004914923 & $3548 \pm 37$ & $1114 \pm 26$ & $3561 \pm 55$ & - \\
KIC012009504 & - & $1139 \pm 29$ & - & $1116 \pm 64$ \\
\hline
\end{tabular}

an efficient exploitation of the seismic data to be obtained by PLATO/ESA [17] for a wide range of main-sequence solar-like stars.

\section{Acknowledgements}

Part of this work had the support of the European Commission under the SPACEINN project (FP7-SPACE-2012-312844) and funds from FCT (UID/FIS/04434/2013 and PTDC/FISAST/7073/2014).

\section{References}

[1] D.O. Gough, M.J. Thompson, Magnetic Perturbations to Stellar Oscillation Eigenfrequencies, in Advances in Helio- and Asteroseismology, edited by J. Christensen-Dalsgaard, S. Frandsen (1988), Vol. 123 of IAU Symposium, p. 155

[2] S.V. Vorontsov, A Search of the Effects of Magnetic Field in the Solar 5-MINUTE Oscillations, in Advances in Helio- and Asteroseismology, edited by J. Christensen-Dalsgaard, S. Frandsen (1988), Vol. 123 of IAU Symposium, p. 151

[3] D.O. Gough, Comments on Helioseismic Inference, in Progress of Seismology of the Sun and Stars, edited by Y. Osaki, H. Shibahashi (1990), Vol. 367 of Lecture Notes in Physics, Berlin Springer Verlag, p. 283

[4] M.J.P.F.G. Monteiro, J. Christensen-Dalsgaard, M.J. Thompson, A\&A 283, 247 (1994)

[5] M.J.P.F.G. Monteiro, M.J. Thompson, MNRAS 361, 1187 (2005)
[6] M.J.P.F.G. Monteiro, J. Christensen-Dalsgaard, M.J. Thompson, MNRAS 316, 165 (2000)

[7] G. Houdek, D.O. Gough, MNRAS 375, 861 (2007)

[8] J.P. Faria, Master's thesis, Masters Degree in Astronomy, Faculty of Sciences - University of Porto (2013), http://hdl . handle.net/10216/69506

[9] A. Mazumdar, M.J.P.F.G. Monteiro, J. Ballot, H.M. Antia, S. Basu, G. Houdek, S. Mathur, M.S. Cunha, V. Silva Aguirre, R.A. García et al., ApJ 782, 18 (2014)

[10] P. Charbonneau, ApJS 101, 309 (1995)

[11] A.M. Broomhall, W.J. Chaplin, G.R. Davies, Y. Elsworth, S.T. Fletcher, S.J. Hale, B. Miller, R. New, MNRAS 396, L100 (2009)

[12] J.P. Marques, M.J.P.F.G. Monteiro, J.M. Fernandes, ApSS 316, 173 (2008)

[13] P. Morel, Y. Lebreton, ApSS 316, 61 (2008)

[14] M.J.P.F.G. Monteiro, ApSS 316, 121 (2008)

[15] K. Verma, J.P. Faria, H.M. Antia, S. Basu, A. Mazumdar, M.J.P.F.G. Monteiro, T. Appourchaux, W.J. Chaplin, R.A. García, T.S. Metcalfe, ApJ 790, 138 (2014)

[16] T. Appourchaux, W.J. Chaplin, R.A. García, M. Gruberbauer, G.A. Verner, H.M. Antia, O. Benomar, T.L. Campante, G.R. Davies, S. Deheuvels et al., A\&A 543, A54 (2012)

[17] H. Rauer, C. Catala, C. Aerts, T. Appourchaux, W. Benz, A. Brandeker, J. Christensen-Dalsgaard, M. Deleuil, L. Gizon, M.J. Goupil et al., Experimental Astronomy 38, 249 (2014) 